\title{
Mediación editorial y valor agregado: La obra de Jaime Saenz desde las redes sociales del pasado
}

\author{
Roberto Pareja
}

Investigador independiente, Séminaire MEDET-LAT

\begin{abstract}
This article offers a critical study of the place that Jaime Saenz occupies within the broader context of translated literatures to shed light on the construction of his image as a writer both in Bolivia and in the countries in which he has been translated. How is Saenz's work viewed from a global perspective? I propose a methodology that analyzes and visualizes web media to study Saenz's position vis-à-vis his Bolivian and Latin American peers within the local and global mediation scene. I read Saenz's work through a web media map that visualizes him diachronically within a framework that considers his production and reception by publishers, editors, translators, literary agents, critics and sponsors, in national and international contexts. In this article I focus on showing the editorial mediation processes of Saenz's work comparatively and in relation not only to literature, but to the broader cultural field, which includes the social sciences and audiovisual arts. The image of a writer that results from this process (dark, accursed, esoteric, and associated with a mystical Andean spirituality) adds symbolic value and prestige to the author. A web media analysis helps to visualize how this added value was created over time and within its socio-political contexts.

\section{Keywords}

Data analysis and visualization, digital humanities, editorial mediation, MLA database, social media of the past, WorldCat database
\end{abstract}




\section{Resumen}

Una mirada crítica al lugar que ocupa Jaime Saenz en el panorama de la traducción de literaturas extranjeras podría clarificar la construcción de su imagen de escritor tanto en Bolivia como en los países en los que se lo ha traducido. ¿Cómo se ve la obra de Saenz desde una perspectiva global? Propongo una metodología de análisis y visualización de redes para estudiar la posición de Saenz vis-à-vis sus pares bolivianos y latinoamericanos dentro del panorama de la mediación local y global. Leo la obra de Saenz a través de un mapa de redes que lo visualiza diacrónicamente dentro de los factores de su producción y su recepción, tales como editoriales, editores, traductores y agentes literarios, críticos y mecenas, en contexto nacional e internacional. En este artículo me concentro en mostrar los procesos de mediación editorial de la obra de Saenz comparativamente y en relación no sólo a la literatura, sino al campo cultural más amplio, que incluye las ciencias sociales y las artes audiovisuales. La imagen de escritor que se construye en este proceso (poeta oscuro, maldito y esotérico, místico de la espiritualidad andina) es un valor simbólico y de prestigio agregado. El análisis de redes ayuda a visualizar cómo se creó ese valor agregado en la obra de Saenz a lo largo del tiempo y en sus contextos sociopolíticos.

\section{Palabras clave}

Análisis y visualización de datos, base de datos MLA, base de datos WorldCat, humanidades digitales, mediación editorial, redes sociales del pasado

El mito que Jaime Saenz (1921-1986) creó con su obra y vida puede ser comparable con lo que ha generado una narrativa de los orígenes del cosmos. Este mito saenceano ha sido interpretado de diversas maneras; por ejemplo, desde la fenomenología, como una "reducción poética" que funda una ontología completa con su estética y su política del lugar (Rivera-Rodas 1983, 1991) y, añadiría, con su genio del lugar que encarna sobre todo en el aparapita, pero que tiene otras encarnaciones como el Choqueyapu o el Illimani. A la luz de este mito, el cargador aymara (el aparapita) lleva la ciudad de La Paz en su bolsa y sobre su espalda, produciéndola como lugar y como sociedad a través de un acto de autonegación ("sacarse el cuerpo"), un sacrificio nihilista envuelto en la alegría apocalíptica del júbilo. Las líneas finales del ensayo "El aparapita de La Paz", publicado en la revista parisina Nuevo Mundo en 1968 dicen:

Ahora bien; por mi parte, en cuanto a mi manera de ver, iqué sé yo! Vaya uno a saber si él no se apodera de la ciudad. Yo quisiera que mis ojos viesen lo que yo veo: es él, asimilándose en un trance ideal pero al mismo tiempo 
no es él, es la ciudad quien se asimila, volviéndose verdadera por la irrupción del indio. Del indio, que en la ciudad se volvió aparapita. (8)

Para Oscar Rivera-Rodas la escritura de Saenz es una poética de la inteligibilidad que se opone a la clausura metafísica por medio de una reducción poética que nos acerca al acto y materialidad originarios inscritos en el lenguaje y los sentidos. El espacio urbano donde este mito se despliega es una La Paz marginal, periférica y nocturna, en la cual el "misticismo telúrico", lo que Guillermo Francovich Ilamó la "mística de la tierra" y que estudió en la obra de Franz Tamayo y otros pensadores letrados de principios del siglo XX (51-58), se transforma en algo diferente aunque todavía claramente relacionado con la tradición estudiada por Francovich. Como indica Erich Fisbach al estudiar el género discursivo del retrato en Saenz, el aparapita ha dejado de ser el estereotipo del indigenismo y se ha convertido en otra cosa, pero aún cumple la función de ser un genio del lugar que reconstruye la totalidad cósmica a partir de fragmentos.

El mito saenceano adquiere así vida propia, separándose de la obra y de la vida del autor. Esto sucede tanto en un contexto extremadamente local como en uno global, sobre todo teniendo en cuenta que Saenz es uno de los escritores bolivianos más publicados fuera de Bolivia (Alarcón 75, n. 3). Este fenómeno se puede estudiar en el desplazamiento que se produce desde la crítica literaria académica hacia la comunicación masiva de ciertos términos e imágenes. La creación de una imagen de autor y su inserción en los mercados editoriales globales produce un valor agregado que es necesario estudiar. Por esta razón, este mito popularizado no es simplemente, como sugiere Gustavo Alarcón, "imagen exagerada" o "lectura errónea y desviada" (Alarcón 75), sino que, de hecho, es parte de un proceso de mediación editorial bastante común, en el cual se toman ciertos rasgos del autor y de la crítica y se los promociona junto a la obra. De ahí que el misticismo y el consumo de alcohol en la obra y en la vida de Saenz se convierten en un imán alrededor del cual giran otros aspectos. Así, se tiene a Alarcón quien indica, apoyándose en un testimonio de Álvaro Díez Astete, que el nazismo "esotérico" como modelo de un "vitalismo telúrico ancestral" atrajo a Saenz sobre todo en la primera parte de su vida, marcada por un viaje a Alemania entre 1938 y 1939, pero perdurará en su interés por la alquimia (75-78) y, se puede añadir, se expresará en el costumbrismo y el nacionalismo de Imágenes paceñas (1979). También podríamos señalar la Introducción de Saenz al libro de Carlos Mesa de 1984 sobre el arquitecto Emilio Villanueva, que no sólo es interesante por la temática "nacionalista" aparente en el título (Emilio Villanueva: hacia una arquitectura 
nacional), sino porque se publica durante el periodo de hiperinflación que terminaría con el gobierno de la UDP (Unidad Democrática Popular) en 1985 y a sólo tres años de la muerte del poeta paceño.

Alba Balderrama, en una reciente entrevista a Luis $\mathrm{H}$. Antezana y Marcelo Paz-Soldán a propósito de la celebración del centenario del nacimiento Saenz, recuenta este mito saenceano a través de la entrevista que le hiciera Antezana en 1978 al escritor paceño (Antezana y Saenz 1978). Las palabras claves que ayudaron a construir el mito de Saenz aparecen a menudo tanto en la entrevista de 1978 como en la de Balderrama a Antezana y Paz-Soldán. En esta última, términos como "mito" o "ser mítico" aparecen junto a expresiones del campo semántico de la filosofía idealista y fenomenológica, tales como "oscuridad de uno mismo" o "misterio del ser", entretejidas con alusiones a la "cuestión hermética" y "la obra" (Balderrama). Estas referencias al misticismo alquímico y a la fusión de subjetividad y materialidad parecen ser rasgos centrales de la obra de Saenz, al menos según una buena parte de la crítica (Rivera-Rodas, Monasterios, Velásquez Guzmán). Rivera-Rodas, uno de los primeros críticos en contribuir a la canonización de la poesía de Saenz, sitúa su práctica poética dentro de la problemática metafísica y fenomenológica de la filosofía occidental y en el contexto de otros poetas bolivianos coetáneos como Óscar Cerruto o Antonio Ávila Jiménez. Para Rivera-Rodas, Saenz es ante todo un poeta de la inteligibilidad que explora la interioridad y la oscuridad a través de la experiencia de la muerte y del sentido del tacto como medio para alcanzar lo inteligible, es decir la experiencia mística de la unión de sujeto que contempla y objeto contemplado (Rivera-Rodas 1991, 165-201). Por su parte Mónica Velásquez Guzmán, al estudiar la imagen de la noche en la obra de Saenz, propone que esta metáfora nocturna y musical

deviene un concepto donde Romanticismo alemán o mundo andino tocan los resquicios de lo sagrado: en este y otros sentidos su obra roza la música como universo central para encarnar el vacío y las tensiones del artista; su lenguaje está anclado en la paradoja como manera de estar en el mundo con el humor suficiente para resistirlo; la presencia-ausencia del dolor (personal y del mundo) diseñan los hitos de un camino místico pero fundamentalmente de reflexión existencial sobre el quehacer poético donde todo, incluso la figura del poeta, son un constructo del lenguaje poético. (39)

Finalmente, y sin ser para nada exhaustivo en esta breve revisión, la narrativa de Saenz, particularmente Felipe Delgado y la figura del aparapita, ha sido estudiada desde distintos ángulos que tienden a converger en la noción de una totalidad construida a partir de fragmentos. Esto se ve, por ejemplo, en los análisis del saco delaparapita (Antezana 1982, 1995; Sanjinés 1994, 2003; 
Wolfenzon 2015) que, a partir de un misticismo de la abyección que mezcla cosmogonía indígena y pensamiento europeo, presentan al aparapita como una condensación de la experiencia urbana subalterna (Sanjinés 1994, 16062), o que lo describen como la encarnación de lo heterogéneo, lo monstruoso y lo abigarrado que dan sentido a la ciudad de La Paz como metrópoli andina (Antezana 1995, 23-29).

¿Hasta qué punto y en qué contextos las ideas y términos que emergen de la crítica literaria se tornan influyentes fuera del campo estrictamente académico? Esta pregunta contiene otras preguntas que nos llevarán a estudiar la mediación editorial de la obra de Saenz y la construcción de su imagen de autor. En Bolivia, la canonización de Saenz y la construcción de su imagen de poeta maldito, místico de la espiritualidad andina e intérprete privilegiado del genio telúrico de la ciudad, se da en una especie de traducción desde la obra misma, y desde los conceptos de la crítica literaria, hacia las artes escénicas y audiovisuales. Ejemplos de ello son la obra teatral No le digas (2007), escrita y actuada por David Mondacca en base a textos de Saenz, que tuvo una amplia circulación dentro y fuera de Bolivia, y el CD-ROM multimedia La Bodega de Jaime Saenz, conceptualizado por Luis H. Antezana y Marcelo Paz-Soldán. La terminología saenceana desborda lo meramente literario para contagiar el lenguaje de otras artes y, finalmente, llegar a los sentidos comunes que circulan en los medios de comunicación de masas.

Fuera de Bolivia, las traducciones al inglés, al alemán y al italiano son ejemplos de la mediación editorial entre lenguas y la consiguiente representación hacia el público de la sociedad local de un producto cultural extranjero. ¿Se traduce también la imagen de poeta místico de la espiritualidad andina? Y si es así ¿de qué manera? ¿O son las traducciones las que contribuyen mayormente a la construcción de esta imagen de autor? En un mapa mayor de la mediación editorial latinoamericana, ¿es el caso de Saenz típico o una anomalía?

Estudiar la red personal de Saenz en el contexto boliviano, latinoamericano y global ayudará a entender la producción de valor (agregado) que se da en la creación de la imagen de autor. De este modo, las relaciones entre autor y editor, autor y traductor, autor y crítico, contenidas en la información bibliográfica, se pueden empezar a reconstruir como redes de coparticipación en proyectos editoriales. 


\section{Metadatos bibliográficos y mediación editorial}

Para empezar a precisar una serie de preguntas sobre este tema, mi análisis de un conjunto de datos extraídos de las bases de datos de la Modern Language Association International Bibliography (MLA) International Bibliography y de WorldCat (el catálogo más grande del mundo), busca entender a Saenz en el contexto de su recepción crítica global, antes que desde un lente estrechamente local. ${ }^{1}$ La base de datos del MLA es muy útil para estudiar la literatura latinoamericana desde una perspectiva global a través de macrodatos, es decir datos que, ya sea por su volumen y/o por su riqueza y complejidad, son plasibles de ser analizados usando métodos computacionales. Esta base de datos, además de extensa, abarca publicaciones de numerosas y variadas revistas académicas en diversas lenguas, desde 1888 hasta nuestros días. Aunque deja de los soportes multimedia (por ejemplo, los CD-ROM) y sólo puede ofrecer una mirada parcial por limitarse a artículos de revistas y algunos capítulos de libros, su enfoque en la literatura secundaria producida en formato de artículo académico genera un corpus de datos suficientemente extenso y complejo para aplicar métodos de lo que Franco Moretti (2013 y 2007) denominó "lectura distante". Las operaciones de "lectura distante" (en tensa relación con la "close reading") deben realizarse dentro de un "espacio documental" formalizado, es decir al interior de una serie de documentos que han sido marcados con esquemas de metadatos estandartizados (Grandjean; Grandjean y Jacomy), como en el caso de las series archivísticas o de los datos bibliográficos. De este modo, la base de datos del MLA se convierte en una fuente imprescindible para estudiar la mediación editorial de la literatura latinoamericana desde una perspectiva global.

Entiendo aquí mediación editorial como la puesta en libro de una obra, pasando por una serie transformaciones que median entre el texto y el producto libro y que presupone un entramado institucional e interpersonal extremadamente complejo: "un dispositivo compuesto de distintos organismos, disciplinas y prácticas" (Guerrero 202). Una de esas transformaciones reside en la construcción de un vocabulario para hablar sobre el autor y su obra, terminología que puede aparecer en los paratextos editoriales, por ejemplo. La mediación editorial se produce a través del vínculo entre dos o más actores que por medio de variados códigos (traducción, 1 Los datos extraídos de ambas bases de datos alcanzan a más de 600 entradas
bibliográficas. 
diseño gráfico, paratextos editoriales) posibilitan el trabajo de presentar y hacer circular las literaturas en un público nacional y global (Pareja 2021).

Una ventaja evidente de trabajar con datos extraídos de la base de datos del MLA es que dispone de un tesauro que no solamente permite obtener conjuntos de datos por países o autores, sino que, como Francisco Espino sugiere en su estudio de la presencia de las literaturas nacionales de América Latina en los datos del MLA, éstos abren "tal cantidad de perspectivas" que se necesitarían sucesivos trabajos de aproximación para poder abarcarlas (80). Espino rastrea, por ejemplo, la excepcionalidad de Cuba dentro del conjunto de datos por su frecuencia de aparición en el corpus, mucho mayor que la de las otras literaturas, y que es excepcional si las comparamos usando un índice basado en la frecuencia de aparición y la población de los respectivos países. La excepcionalidad de Cuba en este contexto se puede atribuir obviamente a varios factores y el estudio de los metadatos bibliográficos abre ventanas para entender estos factores desde distintas perspectivas (Espino 72-80). De manera similar a Espino, pero para un contexto y un caso diferentes, considero que las tablas de frecuencia son centrales en cualquier análisis de este tipo de datos y, dado mi enfoque en la recepción crítica de un autor en el contexto global de una literatura nacional, para mí es esencial saber la frecuencia y distribución de ciertos temas en el conjunto de datos que he extraído. Por otra parte, me concentro en metadatos muy específicos que permiten visualizar los factores de la producción y recepción, tales como autores, editoriales y editores, traductores y agentes literarios, críticos y otros actores. Por ello es necesario discutir brevemente los métodos que usé para la extracción, limpieza y modelización de los datos, particularmente la creación de variables categóricas, inexistentes en los datos originales, que me permitieron desarrollar una taxonomía útil para desagregar los datos temáticos del tesauro del MLA y analizarlos a un nivel de granularidad mayor.

Cada entrada de la base de datos del MLA está acompañada de una serie de temas indexados en un tesauro. Un análisis sumario de esos datos textuales revela una tipología que se puede automatizar usando "expresiones regulares" ("Expresión regular") a través de lenguajes como Python o R y herramientas como OpenRefine (OpenRefine for Social Science Data) para reorganizar los datos formando matrices. De este modo logré crear las categorías de Persona, Obra, Periodo y Tópico, y aplicarlas a los datos para realizar análisis y visualizaciones a través de cada una de estas categorías o una combinación de ellas usando herramientas como el lenguaje $R$ y su ambiente de programación (RStudio). Al aislar la categoría Persona, puedo capturar los 
datos que se refieren a autores estudiados por la crítica, mientras que la categoría Obra refiere a las obras estudiadas. La categoría Periodo, por su parte, representa los periodos históricos cubiertos por los artículos académicos, al tiempo que Tópico cubre términos que abarcan una gran variedad de temas, desde conceptos teórico-metodológicos a terminología literaria básica sobre género textual-discursivo, área geográfico-cultural o disciplina académica.

La ventaja de reorganizar los datos de esta manera es que puedo fácilmente visualizar patrones y formular hipótesis a través de un conjunto de datos interrogables desde distintos ángulos. Si observamos la visualización "Temas por Categoría" (Fig. 1) vemos que, al limitarnos a los 50 temas más frecuentes por categoría, Saenz aparece en la categoría Persona junto a Edmundo-Paz Soldán y Alcides Arguedas, los dos autores más frecuentemente estudiados.

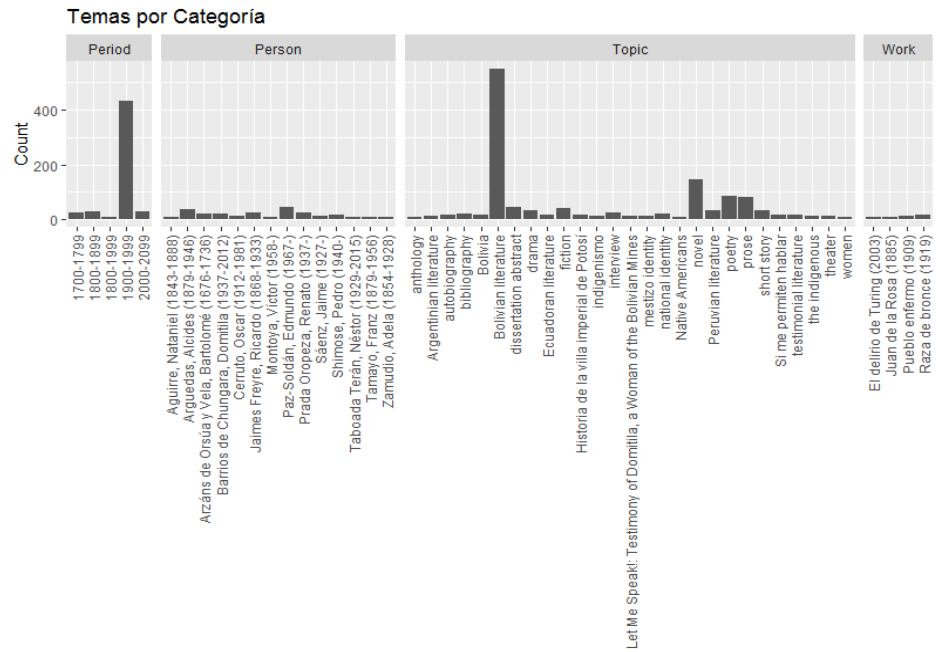

Figura 1: Temas por Categoría

Fuente: Pareja, RPubs, "Literatura_Boliviana_MLA_Temas_por_Categoría" https://rpubs.com/rpareja/739309 
Algo de notar en este panorama es que Paz-Soldán participa por partida doble en este conjunto de datos, ya que aparece como autor de artículos académicos y como autor estudiado. En la visualización antes referida esto no es directamente observable, pero podemos formular la hipótesis de que la sinergia creada entre estas dos dimensiones de Paz-Soldán en los datos de la recepción crítica de la literatura boliviana explica el lugar de Arguedas como segundo autor frecuentemente estudiado, ya que Paz-Soldán, en tanto crítico literario, ha contribuido extensamente a la frecuencia del tema "Arguedas, Alcides (1879-1946)".

Es más que probable que esta sinergia se replique en el caso de Luis $\mathrm{H}$. Antezana y Saenz cuando se haga un relevo confiable de los fondos bibliográficos de los repositorios bolivianos y se enriquezcan los datos iniciales. Antezana ha escrito mucho sobre Saenz y fue probablemente el primero en analizar Felipe Delgado (1979) en un artículo de la revista Hipótesis de 1982 (Antezana, "Sobre Felipe Delgado") que luego aparecería por partes en el periódico Presencia a lo largo del mes de mayo de ese mismo año, momento en que comienza el proceso democrático en Bolivia con el gobierno de la UDP. Muchos de los textos de Antezana, incluyendo los que escribió sobre Saenz, fueron publicados en suplementos literarios de la prensa diaria, en revistas de diversa índole o como prefacios de libros, para no mencionar las publicaciones en formato multimedia. Aunque compilados después en libros, estos artículos de crítica literaria-cultural tienen una trayectoria y circulación diferente que la de artículos publicados en revistas académicas indexadas, razón por la que el MLA no los recoge. También es necesario señalar que, en muchos casos, el tesauro no indexa todos los temas tratados por un artículo, particularmente cuando se trata de un panorama crítico muy amplio como es el caso de algunos de los artículos de Antezana que sí recoge el MLA. Por ejemplo, en el artículo de 1999 "Narrativa y poesía bolivianas (Indicación y Antología), el MLA sólo adjunta temas generales como "Bolivian Literature", el periodo "1900-1999", y los tópicos "Fiction" y "Poetry", pero no los autores incluidos en ese panorama.

Es así que, a pesar de que Antezana sí trata, aunque brevemente, la narrativa de Saenz en el artículo anteriormente citado, el tesauro no nos permite descubrirlo. Es significativo que todos los artículos de Antezana incluidos en la base de datos del MLA son artículos aparecidos en revistas europeas o norteamericanas, dejando de lado, por su misma naturaleza, el extenso corpus de artículos de crítica literaria-cultural aparecidos primero en publicaciones periódicas mayormente de Bolivia y reunidos luego en 
volúmenes como Sentidos Comunes (1995), el cual incluye "La poética del saco de aparapita" publicado primero en el suplemento Presencia Literaria en abril de 1987. Finalmente, hay que mencionar dos artículos indexados por el MLA sobre la narrativa y poesía de Saenz bajo la autoría colectiva de los miembros del Taller Hipótesis-Cochabamba y Taller Hipótesis-La Paz, aparecidos en 1986 en el número 52 de Revista Iberoamericana. Estos dos artículos se pueden asociar claramente a Antezana, puesto que éste era miembro de ese taller de crítica literaria que publicaba en Bolivia la ya mencionada revista Hipótesis. En este contexto, una buena base para iniciar un relevo exhaustivo de la bibliografía saenceana en Bolivia es la tesis de maestría de Rodolfo Ortiz sobre la obra de Saenz, donde se incluye una bibliografía de y sobre Saenz que recopila precisamente, desde lo local, la amplia recepción crítica de la obra de Saenz y, claro, hace visible la continua atención que Antezana le ha brindado a Saenz desde 1977 hasta el presente (Ortiz Oporto 85-100). Por ejemplo, Ortiz recoge el disco multimedia La bodega de Jaime Saenz, pero no se lo atribuye a Antezana ni a Marcelo Paz-Soldán, aunque claramente ambos son los creadores conceptuales del producto multimedia. No incluye, por otra parte, el sitio de internet Jaime Saenz creado por Leonardo García Pabón, un recurso digital que con toda probabilidad ayudó a difundir la obra de Saenz y el mito saenceano, ya que presenta una biografía, una selección de textos y variados recursos audiovisuales descargables, desde grabaciones de una lectura poética de 1972 en las que figuran tanto García Pabón como Luis H. Antezana al lado de Saenz, hasta las obras teatrales de David Mondacca en base a textos de Saenz; las composiciones musicales inspiradas en su obra, fotos, dibujos y tapas de libros (García Pabón 2021). Hay una continuidad de proyecto cultural entre el recurso multimedia de Antezana y Paz-Soldán, el sitio de internet de García Pabón y todos los artistas que han contribuido con sus adaptaciones teatrales, videísticas, musicales o pictóricas, a la difusión de la obra y el mito. Antezana aparece como un actor central en este proceso de mediación editorial de la obra de Saenz en Bolivia y a nivel global, y es una de las piezas claves para reconstruir el mapa de las redes que hicieron posible el mito saenceano; es decir, lo que podríamos llamar condiciones de posibilidad para la producción simbólica alrededor de la obra de Saenz.

Otro actor importante en esta red saenceana es Blanca Wiethtüchter, cuyo estudio "Estructuras de lo imaginario en la obra poética de Jaime Saenz" acompaña la Obra poética de Saenz publicada por la Biblioteca del Sesquicentenario de la República en 1975, en pleno gobierno militar de Hugo Banzer Suárez. Ese estudio es probablemente una versión de la Mémoire de Maîtrise (Structure de l'imaginaire dans l'œuvre poétique de Jaime Saenz), 
defendida ese mismo año en la Universidad de Paris VIII bajo la dirección de Sául Yurkievich. Terniendo en cuenta esta temprana conexión entre la obra poética de Saenz y el espacio académico francés, es interesante que no se lo tradujera al idioma francés, sobre todo considerando que durante esos años en Francia se publicaba mucha poesía latinoamericana en traducción. ¿Quizás la animosidad de Saenz contra el fenómeno del boom literario latinoamericano, difundida en la prensa periódica, tenga que ver con esto? (Saenz 1978). ${ }^{2}$

Las conexiones entre Saenz y Wiethüchter van más allá de la relación entre obra y crítica e incluyen la escritura biográfica (Memoria solicitada); una mutua atención a nivel de escritores a través de la escritura de prólogos, por ejemplo el de Saenz al poemario Asistir al tiempo, reproducido en la traducción al italiano, y el registro en video de adaptaciones teatrales en colaboración con el actor David Mondacca y el compositor Alberto Villalpando ("Habitantes de la ciudad"). Finalmente, Antezana cuenta que fue precisamente Wiethüchter quien le presentó a Saenz para concertar la famosa entrevista de 1978 publicada en Hipótesis (Antezana y Saenz 1978, Balderrama 2021).

Si volvemos a la visualización de los datos del MLA se observa que en el gráfico "Obra por frecuencia" (Fig. 2) la sinergia entre Paz-Soldán y Arguedas puede seguirse en la mayoría de obras estudiadas con mayor frecuencia.

\footnotetext{
2 Johnson and Gander repiten también esta percepción en su introducción a Immanent Visitor: "In the 1960s and 1970s, while his great Bolivian contemporary, Oscar Cerruto, was gaining a reputation for poetry of formal and psychological nuance, and as the global reach of postmodernism was beginning to rush literature into radical new forms, Saenz, stubbornly mystical and baroque, was on his own. His work was certainly innovative, absorbing the fantastic, the psychological, and the symbolic. But it wasn't formally radical enough to situate him among the international avant-garde; it wasn't politically specific enough to find favor with the ascendant literary left, and it was too weird to ride into popularity on the coattails of writers like Cortázar and Vargas Llosa during the Latin American boom of the 1970s" (xii) [En las décadas de 1960 y 1970, mientras su gran contemporáneo boliviano, Oscar Cerruto, ganaba reputación por una poesía de matices formales y psicológicos, y mientras el alcance global del posmodernismo comenzaba a precipitar la literatura hacia formas nuevas y radicales, Saenz, obstinadamente místico y barroco, estaba solo. Su trabajo fue ciertamente innovador, absorbiendo lo fantástico, lo psicológico y lo simbólico. Pero formalmente no era lo suficientemente radical como para ser situardo entre las vanguardias internacionales; desde el punto de vista politico, no era lo suficientemente específico como para encontrar el favor de la izquierda literaria en ascenso, y era demasiado extraño como para ganar popularidad en medio de escritores como Cortázar y Vargas Llosa durante el boom latinoamericano de la década de 1970]. Mi traducción.
} 


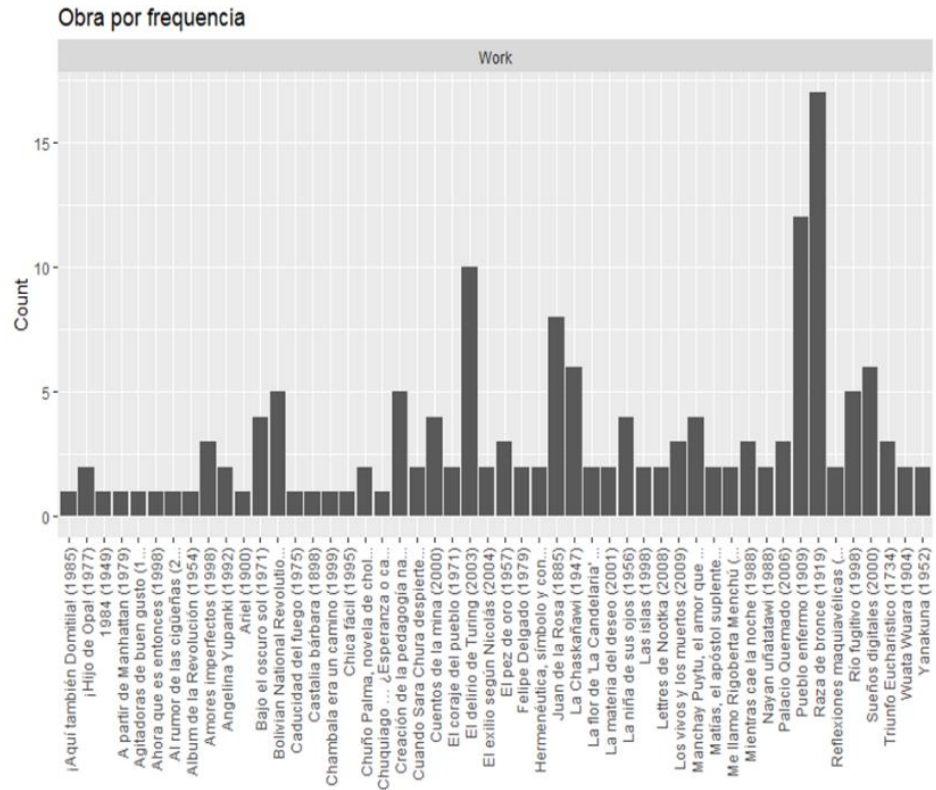

Figura 2: Literatura boliviana. Obra por Frecuencia Fuente: Pareja, RPubs, "MLA_Obra por frecuencia" https://rpubs.com/rpareja/798711

Pueblo enfermo (1909) y Raza de bronce (1919) son las dos obras más estudiadas de todo el corpus, mientras que, por su parte, la cantidad de obras de Paz-Soldán estudiadas sobrepasan con creces a las de Arguedas y, por la frecuencia en que algunas de ellas aparecen en el tesauro del MLA, siguen de cerca las obras de Arguedas. Felipe Delgado (1979) es la única de las obras de Saenz que aparece entre los temas del tesauro, si nos limitamos a las 50 obras más estudiadas, pero se estudia con una frecuencia mucho menor que El delirio de Turing (2003) de Paz-Soldán, por ejemplo. Como dijimos, esta diferencia se podría acortar o remontar drásticamente con la adición de datos relevados de los repositorios bolivianos, además de poder modificar este mapa preliminar enriqueciéndolo con datos para el estudio de las redes locales que hicieron posible el surgimiento del mito saenceano y alentaron su difusión. 
Una mirada-lectura distante permite acercarse a lo nacional desde los intersticios de la juntura entre lo local y lo global. El mito de Saenz, creado probablemente por primera vez para el público general a partir de la entrevista de Antezana a Saenz en 1978, aparece sugerido en los paratextos de las traducciones de su poesía al inglés. Según datos fácilmente consultables en el Virtual International Authority File (VIAF), las publicaciones de y sobre Saenz, crecieron mucho en las dos primeras décadas del siglo XXI y la traducción al inglés de una selección de su poesía es parte de ese pico en la producción editorial de la primera década del siglo. En el prefacio y en la contratapa de Immanent Visitor, la traducción de 2002, los traductores recuentan también el mito y, en una publicación previa de 2001, seleccionan las estrofas III, IV y VI de Recorrer esta distancia para dar una muestra de lo vendría en el volumen del año siguiente. El estoicismo y el estilo epigramático que Franz Tamayo cultivó en las primeras décadas del siglo XX encuentran ecos en estos versos y la traducción al inglés refleja esa actitud que los traductores, siguiendo a García Pabón, asumen como “monástica” (Johnson and Gander xi). García Pabón, poeta y crítico literario, otro de los actores centrales de la red ego-centrada de Saenz, ha difundido, junto a Luis H. Antezana, Elías Blanco Mamani y otros, el vocablo saenceano de "júbilo", una especie de alegría apocalíptica que aparece en este fragmento de la estrofa VI de To Cross This Distance:

In what grows, in what has ceased to grow, in what echoes, in what stays, in what doesn't stay, in the soundless air, in the metamorphosis of the insects, in the murmuring of trees, one can sense the joy of a coming end. (119, mis itálicas)

Escoger a Jaime Saenz para empezar el análisis del conjunto de datos extraídos de la base de datos del MLA es pertinente porque Saenz es un autor prestigioso en los círculos literarios y, como hemos ido viendo, alrededor de su figura se construyó una imagen de autor muy precisa, particularmente mediante dispositivos teatrales y audiovisuales (obras de teatro, multimedia, música, cine), pero también, por supuesto, a través de las traducciones a otras lenguas. La base de datos del MLA me permite, en oposición al close reading, una lectura doblemente "distante" de la recepción crítica y de la mediación editorial, no sólo por la cantidad de datos disponibles sino porque añade una perspectiva global desde el punto de vista de la variedad y extensión de los datos. Y, a pesar de que la literatura boliviana probablemente está 
subrepresentada en los datos del $\mathrm{MLA}^{3}$ éstos son suficientemente representativos para poder realizar un análisis de la recepción crítica global, a contrastarse con lo que queda fuera de ese conjunto de datos y que se puede recuperar del Archivo y Biblioteca Nacionales de Bolivia (ABNB), la Library of Congress (LOC), la Deutsche National Bibliothek, (DNB) o el Catalogo del Servizio Bibliotecario Nazionale (OPAC SBN) de Italia, amén de repositorios de metadatos como WorldCat y VIAF.

Este contraste entre la recepción más local de Saenz y la recepción global(izante) introduce la posibilidad de intervenir en los datos para realizar ciertas operaciones que exploren esa diferencia. Por ejemplo, poder determinar la distribución de ciertos temas por categoría, por extensión temporal o por localización geográfica, son aspectos que posibilitan, a su vez, un análisis con mayor granularidad. La modelización de los datos es fundamental para el análisis de los mismos y para la generación de visualizaciones, ya sean estáticas o interactivas en aplicativos web, para comunicar los conceptos centrales de la investigación a través de macrodatos cuidadosamente curados. El análisis y visualización de metadatos bibliográficos en los Estudios Literarios y Culturales debe verse menos como un producto final o una especie de "prueba" científica y más como una herramienta heurística que permite contrastar presuposiciones críticas y abrir nuevas perspectivas de investigación.

Concretamente, me refiero a la posibilidad de estudiar "las redes sociales del pasado" (Roig-Sanz y Subirana) como una manera de reconstituir la mediación editorial de un periodo determinado. Una posible red ego-centrada de Saenz incluiría elementos de mayor granularidad, que la red que emerge de los meros metadatos bibliográficos no permite visualizar. Podríamos estudiar la asociación entre Saenz y el escritor paceño Franz Tamayo (1879-1956) que, a pesar de no ser su contemporáneo, existe plenamente en la red ego-centrada por virtud de su presencia en los textos mismos, por ejemplo, en los diálogos entre Delgado y Beltrán en la novela Felipe Delgado, un aspecto ya notado por Antezana. Más allá del entorno ego-centrado, y a una escala mayor correspondiente a una red de co-citas de la literatura boliviana, Saenz se

\footnotetext{
3 Espino demuestra que, respecto a su población, las literaturas nacionales de Cuba, Argentina y Chile están sobrerrepresentadas y las de Colombia, Ecuador, Guatemala, México, Perú y Venezuela están subrepresentadas en el corpus del MLA (Espino 73-74). Su selección no incluye Bolivia ni las otras literaturas latinoamericanas, por ejemplo, Brasil. Aunque podemos especular que también Bolivia está subrepresentada, habría que realizar un estudio similar al de Espino para determinarlo comparativamente.
} 
vincularía a autores como René Zavaleta Mercado precisamente a partir de las entidades nombradas en los textos, tales las menciones a Tamayo o a eventos históricos como la Guerra del Chaco (1932-1935) (Antezana 2006, 164-165).

La lectura distante a partir de metadatos y el análisis textual de una obra o un corpus convergen en este tipo de aproximaciones. Un análisis de ese tipo está fuera de los alcances de este trabajo, pero intentaré un acercamiento conceptual y una proyección a futuro para sentar las bases de un potencial análisis y visualización de redes de la mediación editorial de la literatura boliviana, lo cual requiere al mismo tiempo un trabajo de creación de corpus textuales que está por hacerse, aunque precisamente la categorización de los datos textuales extraídos del tesauro MLA es un primer paso. Un segundo paso implicaría el análisis textual computarizado de las obras narrativas de Saenz y de la crítica sobre Saenz usando herramientas de Reconocimiento Óptico de Caracteres (ROC) y aplicando análisis de sentimientos y análisis de redes para clasificar términos y situarlos en sus contextos. El objetivo sería entender la relación entre ciertos nodos de las redes, por ejemplo, la relación entre Saenz y Tamayo desde la ficción novelesca o a partir de la crítica.

Mi punto de partida son en un principio los datos de MLA y, en segundo lugar, datos recuperados de WorldCat. Aunque me limité a ellos para realizar los análisis y las visualizaciones que presento en este trabajo, mostraré avances que ejemplifican la fusión entre el conjunto de datos MLA y de WorldCat y el conjunto de datos extraíbles de las bibliotecas nacionales mencionadas. Por ejemplo, un detalle interesante es que, precisamente por la naturaleza de la base de datos del MLA, el recurso multimedia La bodega de Jaime Saenz ya mencionado, que es central en la recepción de la obra, no aparece indexado por el MLA. Podría haber sido indexado si hubiera sido objeto de algún artículo académico $y$, en ese caso, hubiera sido indexado como uno de los temas del tesauro. La bodega de Jaime Saenz sí aparece, sin embargo, en WorldCat ${ }^{4}$ y en el catálogo de la LOC, pero no en el de ABNB que, además, por varias razones, no me es útil para esta primera fase de mi investigación ${ }^{5}$. Saenz ha sido, sin

\footnotetext{
${ }^{4}$ Aunque se puede encontrar una versión correcta cuando se busca por el nombre del recurso, cuando se busca a través del nombre de autor "Jaime Saenz", WorldCat atribuye este recurso a Edmundo Paz-Soldán y Luis Antezana Ergueta, lo que puede explicar que luego en el fichero de autoridad VIAF correspondiente a Luis H. Antezana este recurso no aparece citado y Marcelo Paz-Soldán no está listado como uno de sus co-autores (ID VIAF: 61590138).

${ }^{5}$ El catálogo en línea del ABNB no es de ayuda por varias razones. En primer lugar, es un catálogo limitado a la Biblioteca Pública, que consiste de una colección para consulta del
} 
embargo, traducido al inglés, al alemán y al italiano, y su obra está bien representada en los repositorios de la LOC, la DNB y la OPAC SBN, por lo que son un buen punto de partida; pero éstos, ni WorldCat, pueden reemplazar un relevamiento de los fondos bibliográficos de $A B N B$, algo que queda para una investigación futura.

\section{Metadatos bibliográficos y recepción crítica}

En principio, me limitaré a delinear los contornos de los datos disponibles en MLA a través de un análisis enfocado en la frecuencia de 4 categorías de temas: Persona, Obra, Periodo y Tópico. La intención es revelar en los datos aquellos tópicos asociados a determinada persona, ya sea autor/a de artículo, o una instancia de los temas, por ejemplo: "Saenz, Jaime, 1921-1987" en la categoría Persona o "poetry" en la categoría Tópico. Con la ayuda de esta taxonomía se pueden realizar otras operaciones en los datos y así generar nuevas perspectivas sobre los datos mismos. Todo esto como un primer paso para empezar a entender la recepción crítica de la obra de Saenz a lo largo del tiempo y como encaja ésta en la mediación editorial de sus textos.

La recepción crítica es parte de la mediación editorial y la base de datos del MLA ayuda precisamente a conectar la literatura secundaria (sea crítica literaria u otro género discursivo) con los procesos de producción editorial que determinan como se fabrican y circulan los libros (incluidos los digitales). Las variables categóricas Persona, Obra, Periodo y Tópico permiten hacer un análisis por frecuencia y así, por ejemplo, podemos ver la frecuencia de una instancia de Persona en los temas, visualizando a los autores más estudiados y ubicando a Saenz en un rango de frecuencia. Lo mismo podemos hacer con las otras categorías; por ejemplo, podemos ver la frecuencia de los tópicos y tener una idea general de la frecuencia con que se estudian ciertos géneros literarios (Pareja, "Literatura_Boliviana_MLA_Temas_por_Categoria"). Incluso se puede crear otra categoría, "Género Textual", que agrupe el variado vocabulario que

\footnotetext{
público lector que no refleja las adquisiciones de la institución. Lo que se denomina Biblioteca Nacional es una colección archivística que no es accesible al público general y que no dispone de un catálogo en línea. Muy probablemente, este recurso multimedia está disponible en los fondos bibliográficos, ya que existe una "Colección: Material No Librario" que lista una signatura topográfica específica para los discos compactos interactivos y multimedia: "SIGNATURA TOPOGRÁFICA: MNL32" ("COLECCIÓN: MATERIAL NO LIBRARIO". Aquí me refiero únicamente al catálogo en línea de la Biblioteca, no al catálogo en línea del Archivo que, por el contrario, sí es muy útil y que ha sido recientemente rediseñado, pero que no es pertinente para esta investigación.
} 
se refiere a lo que, en literatura, se llama "género literario", pero que desborda lo meramente literario e incluye la diferencia ficción/no ficción, el testimonio, el cine o los géneros no-literarios como la bibliografía. Finalmente, podemos establecer los tópicos asociados a determinadas personas en la categoría Persona y así tener una lista de temas asociados a la obra de Saenz (Pareja, "MLA_Saenz en Persona y Tópico") y potencialmente determinar su distribución temporal en base a la fecha de publicación de la literatura secundaria. Discutiremos estos aspectos a continuación, dando ejemplos concretos de los datos y ofreciendo varias visualizaciones que contribuyen a comprender mejor la posición de Saenz dentro de la mediación editorial a nivel nacional y global, y a empezar a reconstituir las redes en que su obra y su imagen de autor emergen como un mito fuertemente asociado a la ciudad de La Paz y sus contextos andinos.

Tal como lo sugieren Antezana y Paz-Soldán en la entrevista de Balderrama ya citada, el fenómeno de la narrativa urbana paceña es amplio e incluye a autores como René Bascopé y Yolanda Bedregal, contemporáneos de Saenz, o Juan Pablo Piñeiro, Adolfo Cárdenas y Wilmer Urrelo, que vienen después y representan una continuación de la poética nocturna y marginal saenceana (Balderrama). En un contexto de mediación editorial es interesante notar que el título de una compilación de narrativa boliviana contemporánea en traducción al inglés sea The Fat Man from La Paz. Contemporary Fiction from Bolivia (Santos) y que tenga en la portada una reproducción de una acuarela de Raúl Lara, cuyo arte se identifica claramente con el mundo nocturno y marginal paceño y con el mito saenceano. Todo esto hace suponer que una categoría tipo "narrativa urbana" podría ser útil para explorar los datos a través de una selección de términos del tesauro MLA. Bajo esta categoría de "narrativa urbana" se encuentran términos relacionados a los siguientes conceptos, entre otros posibles: lo indígena, la fiesta, el alcohol y la música. Saenz se relaciona con otros actores de la red bibliográfica a través de esta categoría en tanto los actores compartan términos del tesauro. "La Paz" sería uno de los términos claves, además de una serie de términos cercanos al concepto de "folclore". También interesa entender como se relacionan los términos taxonómicos y los conceptos en esta red, por ejemplo, entender el aparapita de Saenz como compartiendo con la fiesta del Gran Poder que narrativizan escritores como Cárdenas o Piñeiro, una serie de rasgos que se expresan en los términos seleccionados del tesauro, entre los cuales podrían estar "Andean culture", "folk rituals", el ya mencionado "La Paz" y "the indigenous" (Fig. 3). 


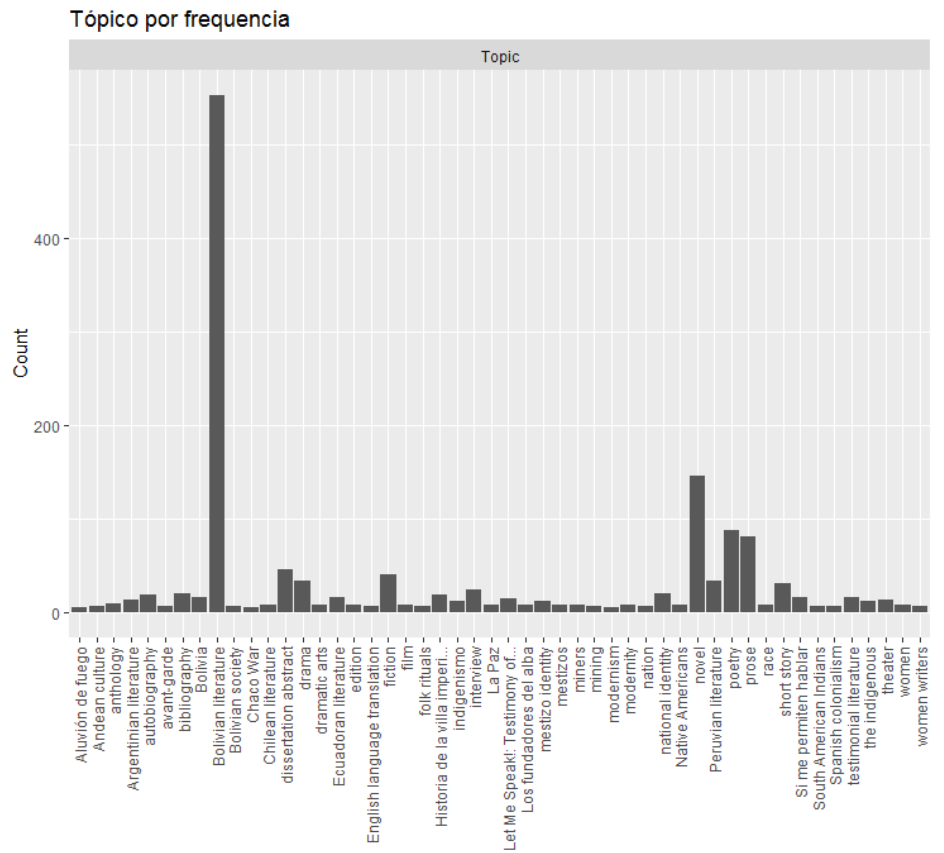

Figura 3: Tópico por frecuencia

Fuente: Pareja, RPubs, “MLA_Literatura Boliviana_Tópico por frecuencia” https://rpubs.com/rpareja/800723

Las conexiones entre actores de la red bibliográfica establecidos de esta forma se pueden convertir en puntas de lanza dirigidas a cuestionar o ayudar a delimitar el conocimiento establecido sobre ciertos temas de la historia cultural, de la sociología y otras disciplinas que estudian la literatura boliviana y latinoamericana. En este caso, las conexiones establecidas en los datos entre Saenz y otros escritores pueden ser usadas como un lente para observar la centralidad de La Paz como nodo conceptual que conecta a una extensa serie de actores, tanto en los datos como en la realidad física, social y simbólica de contextos regionales. Esta centralidad de La Paz se puede observar de forma sistemática desde la perspectiva de la mediación editorial, ya que disponemos de metadatos bibliográficos para construir una red bibliográfica que use "narrativa urbana" como categoría de análisis y como medio de filtrar 
y visualizar los datos. La narrativa urbana paceña podría entonces visualizarse a través de los datos (del MLA, WorldCat, bibliotecas nacionales y otros repositorios) y la presencia de Saenz en esa red sería central. Esta operación aportaría evidencia para sostener que la narrativa urbana paceña es saenceana, apoyando así la hipótesis de Antezana y Paz-Soldán en la varias veces citada entrevista de Balderrama.

\section{Distribución de los temas por categoría}

Para ordenar los temas que el sistema de etiquetas del MLA ofrece fue necesario categorizarlos en 4 áreas: Persona, Obra, Periodo y Tópico. El tesauro atribuye a cada entrada una serie de temas sin categorizar (ver visualización semi-interactiva en Pareja, "Bolivian_Literature_MLA_Subjects"). Para tener una perspectiva más clara sobre esta serie, tuve que introducir una variable categórica que clasificara cada tema. El análisis de la frecuencia de los temas y su distribución por categoría nos permite una primera mirada estructurada a los datos. En la visualización "Temas por categoría", como ya indiqué, el autor que aparece con mayor frecuencia es Edmundo Paz-Soldán, seguido de Alcides Arguedas. Jaime Saenz aparece en el mismo rango que Oscar Cerruto y podemos conjeturar que quizás ambos autores son estudiados en conjunto en uno o varios artículos y que se ha mostrado interés por la poesía de Saenz en relación a otros escritores. Esto se puede observar indirecta y provisionalmente en una visualización de la frecuencia en que la instancia "Saenz, Jaime, 1921-1987"6 aparece junto a otras instancias de Persona estudiadas conjuntamente e instancias de Tópico en la meta-categoría Temas del esquema bibliográfico (Pareja, "MLA_Saenz en Persona y Tópico"). Por ejemplo, podemos observar que la obra de Saenz se estudia en conjunto con la obra de otros escritores bolivianos, como Oscar Cerruto, Pedro Shimose y Jesús Urzagasti.

Título, Autor, Editorial, Lugar, Fecha y, en el caso de los datos de WorldCat, también Tipo de Recurso (impreso, microficha, digital, o audiovisual), son campos del esquema bibliográfico de los datos recuperados de WorldCat interesantes para analizar la presencia de ciertos recursos digitales en las bibliotecas socias de WorldCat y observar si hay relación entre

\footnotetext{
6 Nótese que las visualizaciones que presento aquí tienen esta instancia escrita incorrectamente, como "Saenz, Jaime, 1927-)". Es un error que proviene de los datos recuperados y que dejé intencionalmente sin corregir para resaltar la importancia del proceso de limpieza y modelización.
} 
la frecuencia en que esos recursos aparecen (Pareja, "WorldCat_Saenz por Título y tipo de recurso") y potencialmente dónde aparecen y en qué periodo cronológico. Teniendo en cuenta el concepto de mediación editorial, podríamos preguntarnos si hay algún periodo histórico que muestre un pico en la adopción de ciertos recursos y si la historia cultural puede proveer una manera de entender ese aspecto de los datos, y si lo aprendido en ellos da pistas para entender mejor problemas específicos de la historia cultural, en este caso, la recepción crítica de la obra saenceana a través de datos globales y herramientas digitales para su estudio. Este modelo se puede generalizar para el estudio de la recepción de la literatura latinoamericana en Estados Unidos, incluyendo tanto la literatura secundaria como las traducciones al inglés norteamericano del español latinoamericano y del portugués de Brasil.

En este contexto de análisis de la mediación editorial, los datos recuperados de WorldCat y el MLA se complementan muy bien. El tesauro MLA provee una gran riqueza de vocablos para manejar los datos. Creando taxonomías adhoc para aumentar la capacidad granular sin perder la función abstractiva del modelo, he detectado ciertos patrones textuales para crear categorías que me permitan extraer y usar analíticamente la información contenida en los campos provistos originalmente por el esquema MLA. El conjunto de datos recuperados de WorldCat, por el contrario, no tiene un campo que recoja los temas asociados a cada ítem del catálogo, no al menos en la versión abierta y gratuita del catálogo que usé para este primer acercamiento a las redes de la mediación editorial en el contexto global y local de la literatura boliviana. Los datos recuperados en esta ocasión de WorldCat, sin embargo, tienen campos muy útiles (como Lengua o el ya mencionado Tipo de Recurso) para acercarse a la mediación de la literatura boliviana desde una perspectiva global enfocada en las traducciones de las obras a otras lenguas para estudiar la mediación editorial de las traducciones de literatura (Pareja, "WorldCat_Saenz as Author by Title and Language"; "WorldCat_Obras de Saenz por lengua"). De nuevo, la pregunta quizás más obvia es ¿por qué Saenz no ha sido traducido al francés, una de las lenguas a las que se ha traducido y se sigue traduciendo mucha literatura latinoamericana y que en 1978, cuando Saenz declaraba "[N]o leeré ni a bala a García Márquez, Vargas Llosa ni a Cortázar" (1978), ya había posicionado a París como una de las mecas de la traducción literaria y centro del boom literario latinoamericano. Menos obvio y quizás más interesante es preguntar por las redes que hay detrás de esta específica configuración bibliográfica e introducir la variable temporal. Es decir, preguntar por los actores involucrados en las traducciones y sus relaciones a lo largo del tiempo. 
La perspectiva de WorldCat es la de los catálogos de las bibliotecas nacionales y universitarias y, por lo tanto, la granularidad que proveen sus datos no abarca el contenido específico de los artículos contenidos en las revistas académicas. Para ello existen bases de datos especializadas, como la del MLA. En este sentido, un reto pendiente para mí es acercarme a los datos ofrecidos por los indexadores que tienen una perspectiva latinoamericana, como por ejemplo Redalyc (Sistema de Información Científica Redalyc, Red de Revistas Científicas). WorldCat es una fuente de datos útil para visualizar, después de modelizar y tratar los datos, y aunque sea preliminarmente, la colaboración que se produce entre los actores de la red analizando la frecuencia en la que autores, traductores y otros colaboradores aparecen en la misma publicación. En la nube de palabras de Autores por Frecuencia del corpus WorldCat (Pareja, "WorldCat_Saenz y colaboradores"), podemos observar que Saenz (o la obra de Saenz a través de sus representantes legales, en el caso de las traducciones, o las nuevas ediciones, por ejemplo) aparece colaborando de distintas maneras con una diversidad de actores, individuales y colectivos. Es interesante descubrir un actor colectivo denominado "Archivo Jaime Saenz" en la edición española de La piedra imán de 2018, lo que nos lleva a su vez a preguntar por los albaceas literarios de Saenz y qué rol cumplen en este proceso de mediación editorial que es la traducción y la publicación de su obra en tres lenguas: el alemán, el italiano y el inglés. La función de Antezana y García Pabón en las traducciones al inglés aparece en los paratextos editoriales, ya que ambos son autoridades a las que los traductores acuden para apoyar ciertas decisiones de estilo, por ejemplo. El análisis podría seguir con el rol de Wiethüchter en la red ego-centrada de Saenz filtrando y analizando las ocasiones en que ella y Saenz están en la misma instancia de la categoría Autor (Pareja, "WorldCat_Wiethüchter y Saenz por Título") para visualizar las colaboraciones entre estos dos actores, particularmente en la colaboración post-mortem, es decir en la probable función de albacea literaria de Wiethüchter.

¿Cómo se ve la "imantación" saenceana desde un análisis de los metadatos bibliográficos y de las redes que se tejen desde allí? Es interesante que La piedra imán y Felipe Delgado aparezcan al centro de esta visualización del corpus WorldCat como las obras que condensan la atracción del nombre, la figura y la obra de Saenz desde la perspectiva de su presencia global en los repositorios bibliográficos (Pareja, "WorldCat_Saenz por frecuencia de Título"), pero no es menos notable que, como ya vimos, varias obras de Saenz se encuentren disponibles en formato digital en algunas bibliotecas. 
Un mapa más amplio de actores individuales y colectivos que participan en la mediación editorial de la obra de Saenz puede ser detectado en las redes bibliográficas a partir de análisis y visualizaciones. Saber quiénes son y cómo entran en contacto estos actores puede permitir conectar la red ego-centrada de Saenz a otras redes bibliográficas de autor a nivel nacional, latinoamericano y mundial. Por ejemplo, se puede analizar la presencia de Saenz en otras lenguas en contexto comparativo a nivel nacional y latinoamericano. $Y$ no se trata de hacer un simple ranking, aunque no hay nada malo en eso. Lo que entra en juego en la visualización de redes bibliográficas es una forma de acercarse a las condiciones de recepción de una obra para averiguar si existen redes que la recogen y la presentan de cierta forma al público.

El rol de la crítica literaria en la prensa diaria y las revistas es importante en la creación de una imagen de autor. Como hemos visto, el dispositivo cultural en las condiciones de recepción de la obra de Saenz es amplio e incluye las artes escénico-teatrales y las audiovisuales, además de una novedosa puesta en escena editorial a través de dispositivos multimedia. En este escenario la mediación editorial es fundamental porque incluye vastos espacios informacionales que ligan bibliotecas y archivos de todo tipo, cuyos recursos digitales, como espero haber mostrado, se pueden explorar y usar con provecho.

La pregunta previa de si la mediación editorial (i.e. el paso del texto al libro publicado, distribuido y consumido) en otras lenguas ha influido en la construcción de la imagen de autor o se ha contentado con repetir lo que, por ejemplo, la crítica bolivian(ista) y latinoamerican(ista) ya ha establecido sobre el autor puede empezar a responderse enfocándonos en el caso de la traducción al inglés de la poesía de Saenz. En particular, destaca la influencia de Antezana y García Pabón en la creación de una imagen de autor, y por lo tanto la producción de valor agregado a partir de los paratextos editoriales.

La recepción de Saenz desde la crítica literaria enfocada en la poesía hispanoamericana y caracterizada por un enfoque teórico-metodológico que unifica el campo (fenomenología, hermenéutica, deconstrucción) presupone redes de críticos e instituciones que definen la aceptación de autores en el canon literario. La distribución geográfica de esas redes es importante y debe ser tomada en cuenta. Viendo el caso de Saenz de forma aislada, se observa fácilmente que las redes de críticos y otros agentes se concentran institucionalmente en ciudades como La Paz y Cochabamba, en Bolivia, y en varias universidades, bibliotecas y editoriales en España, Estados Unidos, Italia y Alemania. Si filtraamos el corpus MLA por Autor (de artículo) seleccionando la instancia "poetry" de la categoría Tópico podemos visualizar los críticos 
literarios que, en el contexto de la recepción global de la literatura boliviana, se ocupan de estudiar poesía (Figs. 4 y 5 ).

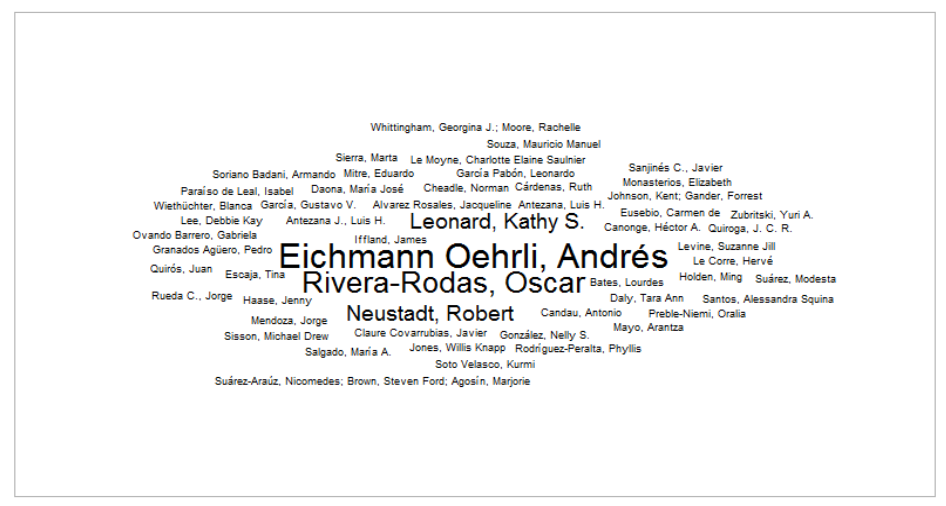

Figura 4: Autor de artículos sobre poesía Fuente: Pareja, RPubs, "MLA_Author_Poetry" https://rpubs.com/rpareja/749875

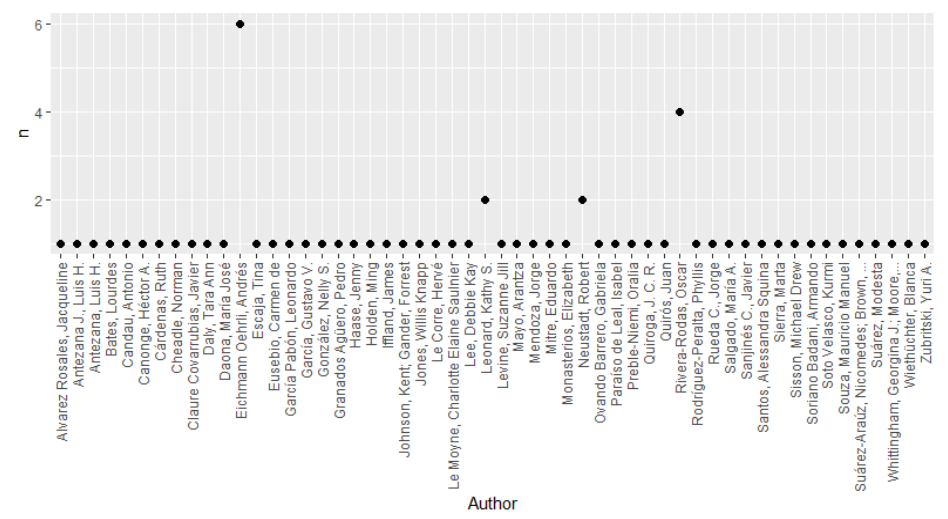

Figura 5: Autor de artículos sobre poesía Fuente: Pareja, RPubs, "MLA_Author_Poetry_geom_point" https://rpubs.com/rpareja/749879 
Inmediatamente notamos que Oscar Rivera-Rodas sobresale entre los críticos, y si vamos a una visualización filtrando los artículos por "poetry" en Tópico y "Saenz, Jaime (1921-1987)" en Persona (Pareja, "MLA_Author by Poetry and Saenz"), descubrimos algunos de los otros tópicos que acompañan a "poetry" en cada ocurrencia donde Saenz es una instancia de Persona: "the indigenous", "senses", "Muerte por el tacto", "nationalism" y "space". Es precisamente Rivera-Rodas uno de los críticos, junto a Wiethüchter, que estudió la obra poética de Saenz desde muy temprano y elaboró un vocabulario con el cual se empezó a entender el discurso saenceano. No vemos a RiveraRodas participando directamente en la mediación editorial, como sí es el caso de Wiethüchter, Antezana y García Pabón, pero la terminología que creó influyó decisivamente en la mediación editorial.

\section{Conclusión}

Una extensión del corpus complejizará esta primera aproximación al incorporar datos provenientes, por ejemplo, de Redylac, de un relevamiento de los fondos de la ABNB o de una nueva extracción de datos del MLA y WorldCat, sin embargo, ya estamos en condiciones de confirmar la utilidad de una lectura distante de la mediación editorial de la literatura boliviana. Por una parte, la función heurística de la lectura distante a través de metadatos bibliográficos quedó en evidencia al bosquejarse una versión de la red egocentrada de Saenz y la función de ésta en la construcción de una imagen de autor que produce valor agregado al inscribirse en la circulación global del mercado editorial a través de ediciones, traducciones e inclusión en antologías. Por otra parte, las fisuras de la juntura entre lo global y lo local se manifestaron en la diferencia de circulación de la crítica sobre el autor, especialmente en el caso de Antezana. Las bases de datos traen sus propias perspectivas geopolíticas y un análisis de la mediación editorial debe ser consciente de estas diferencias.

Finalmente, además de su utilidad heurística en la investigación literaria, los metadatos bibliográficos son fundamentales para la organización del patrimonio cultural en el dominio digital. La reutilización de metadatos a través de su modelización, análisis y visualización constituye una base fundamental para dar acceso a una enorme riqueza documental tanto a los investigadores como al público en general. Tanto la taxonomía creada ad-hoc para esta investigación como mi propuesta de construir la categoría "narrativa urbana (paceña)" para filtrar los datos usando el tesauro disponible en la base de datos de MLA, son ejemplos de herramientas digitales aplicadas a la 
investigación de la mediación editorial y, al mismo tiempo, ejemplos de su potencial aplicación al ámbito de la organización de la información sobre el patrimonio cultural boliviano y latinoamericano.

\section{Bibliografía Citada}

ALARCÓN, Gustavo. 2011. “Jaime Saenz: evocación y revelación”. Rassegna iberistica 93. 75-79.

ANTEZANA, Luis H. 2006. "Zavaleta leyendo Felipe Delgado". René Zavaleta Mercado, ensayos, testimonios, y re-visiones. Maya Aguiluz Ibargüen y Norma de los Ríos Méndez, coords. México: FLACSO, UNAM, CIDESUMSA. 163-170.

---. $\quad$ y Marcelo Paz Soldán. 2005. La bodega de Jaime Saenz. Fundación Simón I. Patiño: CESU-UMSS. Cochabamba: Editorial Nuevo Milenio.

---. 1999. "Narrativa y poesía bolivianas (Indicación y Antología)". Caravelle (1988-) 72. 145-97. [https://www. persee.fr/doc/carav_11476753_1999_num_72_1_2843] página descargada el 30 de agostō, 2021.

---. 1995. "La poética del saco de aparapita". Sentidos Comunes: ensayos y lecturas. Cochabamba: CESU, FACES, UMSS 23-29.

---. $\quad$ 1982. "Sobre Felipe Delgado". Hipótesis. Revista Boliviana de Literatura 13. Reimpreso en Ensayos y Lecturas. La Paz: Ediciones Altiplano, 1986. 333-354.

---. $\quad$ y Jaime Saenz. 1978. "En torno a la obra. Diálogo". Hipótesis. Revista Boliviana de Literatra 10. 169-192.

BALDERRAMA, Alba. 2021. "El campeón del mundo". Opinión. [https://www.opinion.com.bo/articulo/ramona/campeonmundo/20210220004136808560.html] página descargada el 30 de agosto, 2021.

BLANCO MAMANI, Elías. 1998. Jaime Sáenz, el ángel solitario y jubiloso de la noche: Apuntes para una historia de vida. La Paz: Gobierno Municipal de La Paz.

COLECCIÓN: MATERIAL NO LIBRARIO. Archivo y Biblioteca nacionales de Bolivia.

[https://www.archivoybibliotecanacionales.org.bo/index.php?searchw ord=COLECCI\%C3\%93N:\%20MATERIAL\%20NO\%20LIBRARIO\&ordering= newest\&searchphrase=all\&option=com_search] página descargada el 30 de agosto, 2021.

ESPINO, Francisco. 2020. "Big data, criticometría y el estudio de las literaturas nacionales en la bibliografía crítica: el caso excepcional de la literatura cubana". Revista de Humanidades Digitales 5. 66-85.

[https://doi.org/10.5944/rhd.vol.5.2020.27625] página descargada el 30 de agosto 2021. 
"Expresión regular". Wikipedia, la enciclopedia libre.

[https://es.wikipedia.org/w/index.php?title=Expresi\%C3\%B3n_regular \&oldid=134224430] página descargada el 30 de agosto 2021.

FISBACH Erich. 2013. "Dénuement du portrait et portrait du dénuement dans 'El aparapita de La Paz' de Jaime Saenz". Le portrait: Champ d'expérimentation. Fernando Copello y Aurora Delgado-Richet, eds. Rennes: Presses universitaires de Rennes.

COPELLO F, DELGADO-RICHET A. Le portrait: Champ d'expérimentation. Rennes : Presses Universitaires de Rennes, 2013. ISBN : 978-2-75352785-0.

FRANCOVICH, Guillermo. 1956. El pensamiento boliviano en el siglo XX. México: Fondo de Cultura Económica.

GARCÍA PABÓN, Leonardo. 2021. Jaime Saenz. [https://darkwing.uoregon.edu/ /garcia/Saenz/Saenz.html] página descargada el 30 de agosto, 2021.

GRANDJEAN, Martin. y Mathieu Jacomy. 2019. "Translating Networks: Assessing Correspondence Between Network Visualisation and Analytics". Digital Humanities. Utrecht, Netherlands. [https://reticular.hypotheses.org/files/2020/06/Grandjean-Jacomy2019-Translating-Networks.pdf] página descargada el 30 de agosto, 2021.

---. $\quad$ 2016. "Archives Distant Reading: Mapping the Activity of the League of Nations' Intellectual Cooperation". Digital Humanities. Krakow.

GUERRERO, Gustavo. 2018. "La Croix du Sud (1945-1970): Génesis y contextos de la primera colección francesa de literatura latinoamericana". Re-Mapping World Literature: Writing, Book Markets and Epistemologies between Latin America and the Global South / Escrituras, mercados y epistemologías entre América Latina y el Sur Global. Gesine Müller, Jorge J. Locane and Benjamin Loy, eds. Berlin, Boston: De Gruyter. 199-208. [https://doi.org/10.1515/9783110549577-013] Página descargada el 30 de agosto 2021.

JOHNSON, Kent, and Forrest Gander. 2002. "By Way of Introduction". Immanent Visitor: Selected Poems of Jaime Saenz. Berkeley: University of California Press. xi-xvii.

MESA Gisbert, Carlos D., y Jaime Saenz. 1984. Emilio Villanueva: hacia una arquitectura nacional, con 111 ilustraciones. La Paz: Escuela Don Bosco.

"MLA International Bibliography". EBSCO Information Services, Inc.

MONASTERIOS, Elizabeth. 2001. Dilemas de la poesía latinoamericana de fin de siglo: José Emilio Pacheco y Jaime Saenz. La Paz: Plural Editores.

MONDACCA, David, y Jaime Saenz. 2007. No le digas: Guión de teatro en base a textos de Vidas y muertes, Piedra imán, Felipe Delgado y fragmentos de la poesía de Jaime Saenz. La Paz/Cochabamba: Nuevo Milenio, Gente Común.

MORETTI, Franco. 2013. Distant Reading. London: Verso.

---. $\quad$ 2007. Graphs, Maps, Trees: Abstract Models for a Literary History. London: Verso. 
OpenRefine for Social Science Data. https://datacarpentry.org/openrefinesocialsci/. Consultado el 10 de mayo, 2020.

Openrefine.github.com. http://openrefine.org/. Consultado el 26 de abril, 2019.

ORTIZ OPORTO, Rodolfo. 2011. Un impulso irrefrenable me impulsa a escribir lo que escribo: Lecturas de Jaime Saenz. Tesis de Maestría. Universidad Mayor de San Andrés, La Paz.

[http://repositorio.umsa.bo/xmlui/handle/123456789/11384] página descargada el 30 de agosto, 2021.

PAREJA, Roberto. 2021. "La mediación editorial de la literatura latinoamericana en Francia, 1945-2000: Un modelo de datos para el análisis y la visualización de redes". La literatura latinoamericana en versión francesa/La littérature latino-américaine en version française. Gustavo Guerrero and Gersende Camenen. Berlin, Boston: De Gruyter. 299-330.

---. “Bolivian_Literature_MLA_Subjects". RPubs. [https://rpubs.com/rpareja/737938] página descargada el 30 de agosto, 2021.

---. “Literatura_Boliviana_MLA_Temas_por_Categoria”. RPubs. [https://rpubs.com/rpareja/739309] página descargada el 30 de agosto, 2021.

---. "MLA_Author_Poetry". RPubs. [https://rpubs.com/rpareja/749875] página descargada el 30 de agosto, 2021.

---. “MLA_Author_Poetry_geom_point”. RPubs. [https://rpubs.com/rpareja/749879] página descargada el 30 de agosto. 2021.

---. “MLA_Literatura Boliviana_Tópico por Frecuencia”. RPbs. [https://rpubs.com/rpareja/800723] página descargada el 30 de agosto, 2021.

---. "MLA_Obra por frecuencia". RPubs. [https://rpubs.com/rpareja/798711] página descargada el 30 de agosto, 2021.

---. “MLA_Saenz en Persona y Tópico". RPubs. https://rpubs.com/rpareja/749853 . Consultado el 5 de abril de 2021.

---. "WorldCat_Saenz y colaboradores". RPubs. [https://rpubs.com/rpareja/749829] página descargada el 30 de agosto, 2021.

---. “WorldCat_Obras de Saenz por lengua”. RPubs. [https://rpubs.com/rpareja/749845] página descargada el 30 de agosto, 2021.

---. "WorldCat_Saenz as Author by Title and Language". RPubs. [https://rpubs.com/rpareja/749841] página descargada el 30 de agosto, 2021.

---. "WorldCat_Saenz por frecuencia de Título". RPubs. [https://rpubs.com/rpareja/749867] página descargada el 30 de agosto, 2021. 
---. "WorldCat_Saenz por Título y tipo de recurso". RPubs. [https://rpubs.com/rpareja/749860] página descargada el 30 de agosto, 2021.

---. "WorldCat_Wiethüchter y Saenz por Título". RPubs. [https://rpubs.com/rpareja/749836] página descargada el 30 de agosto, 2021.

RIVERA-RODAS, Óscar. 1991. La modernidad y sus hermenéuticas poéticas. Poesía boliviana del siglo xx. La Paz: Ediciones Signo.

---. $\quad$ 1983. "La poesía de Jaime Saenz". INTI: Revista de literatura hispánica 18. 59-82. [https://digitalcommons.providence.edu/inti/vol1/iss18/7/] página descargada el 30 de agosto, 2021.

ROIG-SANZ, Diana y Jaume Subirana, eds. 2020. Cultural Organizations, Networks and Mediators in Contemporary Ibero-America. New York: Routledge.

RStudio. rstudio.com [https://rstudio.com/] página descargada el 30 de agosto, 2021.

SANTOS, Rosario, ed. 2000. The Fat Man from La Paz: Contemporary Fiction from Bolivia. Seven Stories Press.

SAENZ, Jaime. 2007. The Night. Forrest Gander and Kent Johnson, trans. Princeton: Princeton University Press.

---. 2002. Immanent Visitor: Selected Poems of Jaime Saenz. Kent Johnson and Forrest Gander, trans. Berkeley: University of California Press.

---. 2001. "From 'To Cross This Distance'”. Forrest Gander y Kent Johnson, trans. BOMB. 74. 118-119. [https://bombmagazine.org/articles/tocross-this-distance] página descargada el 30 de agosto, 2021.

---. $\quad$ 1999. "Jaime Saenz - five poems from As the Comet Passes". Jacket \# 8. Kent Johnson and Forrest Gander, trans.

[http://jacketmagazine.com/08/saenz.html] Página descargada el 30 de agosto 2021.

---. 1978. “No leeré ni a bala a García Márquez, Vargas Llosa ni a Cortázar: Jaime Saenz". Entrevista por Mery Saavedra. Hoy (La Paz), 10 de diciembre. Reimpresa en Presencia Literaria (domingo 23 de agosto, 1992).

---. $\quad$ 1968. "El aparapita de La Paz". Nuevo Mundo. 26-27. 4-8.

SANJINÉS, Javier. 2003. "A Phenomenological Reading of the Andes: Toward the Desublimation of the Cholo." Guttorm Fløistad, ed. Contemporary philosophy. 8. Philosophy of Latin America. Dordrecht : Kluwer Academic. 171-185.

---. $\quad$ 1994. "Subalternity and the Articulation of Culture in the Bolivian Andes". Dispositio 19 (46): 147-164.

SÉMINAIRE MEDET LAT - Accueil Project. [https://www.projet-medetlat.com] página descargada el 30 de agosto, 2021.

VELÁSQUEZ GUZMÁN, Mónica. 2011. “Re-pensar la poesía contemporánea en Bolivia a través de Saenz, Cerruto, Camargo y Wiethüchter".

Hispamérica 40 (120): 37-46. 
VIRTUAL INTERNATIONAL AUTHORITY FILE (VIAF) ID 61590138. [https://viaf.org/viaf/61590138] página descargada el 30 de agosto, 2021.

WIETHÜCHTER, Blanca. “Habitantes de la ciudad”. Jaime Saenz (1921-1986). [https://darkwing.uoregon.edu/ Igarcia/Saenz/Habitantes.htm] página descargada el 30 de agosto, 2021.

---. 1975. "Estructuras de lo imaginario en la obra poética de Jaime Saenz". Jaime Saenz, Obra Poética. La Paz: Biblioteca del Sesquicentenario de la República. 267-425.

WOLFENZON, Carolyn. 2015. "La figura del aparapita en Felipe Delgado de Jaime Sáenz: nacionalismo, misticismo y conflicto social". Chasqui 44 (2): 241-257.

\section{ULLS}

New articles in this journal are licensed under a Creative Commons Attribution 4.0 United States License.

This journal is published by the University Library System of the University of Pittsburgh as part of its D-Scribe Digital Publishing Program, and is cosponsored by the University of Pittsburgh Press. 